\title{
On Arithmetic Average Fusion and Its Application for Distributed Multi-Bernoulli Multitarget Tracking
}

\author{
Tiancheng Li, Xiaoxu Wang, Yan Liang and Quan Pan
}

\begin{abstract}
Recently, the simple arithmetic averages (AA) fusion has demonstrated promising, even surprising, performance for multitarget information fusion. In this paper, we first analyze the conservativeness and Fréchet mean properties of it, presenting new empirical analysis based on a comprehensive literature review. Then, we propose a target-wise fusion principle for tailoring the AA fusion to accommodate the multi-Bernoulli (MB) process, in which only significant Bernoulli components, each represented by an individual Gaussian mixture, are disseminated and fused in a Bernoulli-to-Bernoulli (B2B) manner. For internode communication, both the consensus and flooding schemes are investigated, respectively. At the core of the proposed MB fusion algorithms, Bernoulli components obtained at different sensors are associated via either clustering or pairwise assignment so that the MB fusion problem is decomposed to parallel B2B fusion subproblems, each resolved via exact Bernoulli-AA fusion. Two communicatively and computationally efficient cardinality consensus approaches are also presented which merely disseminate and fuse target existence probabilities among local MB filters. The accuracy and computing and communication cost of these four approaches are tested in two large scale scenarios with different sensor networks and target trajectories.
\end{abstract}

Index Terms-Target tracking, multisensor fusion, arithmetic average fusion, multi-Bernoulli filter, Fréchet mean, average consensus, cardinality consensus, flooding, clustering.

\section{INTRODUCTION}

D ETECTING and tracking an unknown and time varying number of targets in the cluttered and noisy background, namely multitarget detection and tracking (MTDT), has led to substantial interest in many realms [1]-[3]. Random finite set (RFS) has identified a natural and promising tool for modeling the problem of MTDT in the Markov-Bayes optimal paradigm [4], [5]. The two well established branches of RFS filters are the (cardinalized) probability hypothesis density (PHD) filters [6]-[9] and the multi-Bernoulli (MB) filters [4, Ch.17] [10][13]. In particular, the MB filter models the set of independent targets by a union of independent Bernoulli RFSs. Unlike the (cardinalized) PHD filters which propagate moments (and cardinality distributions), the MB filter propagates the parameters of a $\mathrm{MB}$ distribution that approximate the posterior multitarget density by maintaining over time a number of Bernoulli components (BCs), each corresponding to a potential

This work was partially supported by Key Laboratory Foundation of National Defence Technology under Grant 61424010306 and by National Natural Science Foundation of China under grants 61790552, 61873205, 61873208 and 61672431.

T. Li, X. Wang, Y. Liang and Q. Pan are all with the Key Lab of Information Fusion Technology (Ministry of Education), School of Automation, Northwestern Polytechnical University, Xi' an 710129, China, e-mail: \{t.c.li,woyaofly1982,liangyan,quanpan\}@nwpu.edu.cn

Preprint. DOI: $10.36227 /$ techrxiv.11599902 target. It has the same complexity as the PHD filter, which scales linearly in the number of measurements.

When a sensor network is involved, a significant scientific problem arises as known as multisensor data fusion of which the key challenges are twofold. First, information correlation between sensors is ubiquitous which are often unknown and rules out the Bayesian optimal solution [14], [15]. Second, the update process of the Bayes-optimal multisensor RFS filters involves partitioning all of the sensor measurements into disjoint subsets and are computationally intractable even for a few sensors; see the analysis given in [5, Ch.10] [16]. Overload of fusion computation will lead to a delay to local filters and is therefore practically unaffordable in real-time filter implementations.

Both problems become more complicated with the increase of the network size as more sensors typically indicate more complicated internode cross-correlation and higher computation requirement. Simply, more is different. A method works for a few sensors may not necessarily work for massive sensors. Instead of making further efforts to approach the actually impossible Bayes-optimal fusion, it is of high realistic significance to resort to a fusion rule that is

- Real-time: computationally efficient or able to be performed in parallel to the filtering calculation [17], [18],

- Distributed: scalable with regard to the addition or removement of sensors and insensitive to the failure/fault of any single sensor, and

- Conservative: immune to the unknown, perhaps timevarying, internode cross-correlation [19], [20].

These requirements give rise to the use of average consensus approaches [21]-[24] for distributed information fusion over large scale sensor networks and has been proven robust, promising in various applications. The two most well-known types of "average" are the linear arithmetic average (AA) and the log-linear geometric average (GA) [25].

The combination of the average consensus approach with the RFS filters originates from the log-linear GA fusion, namely generalized covariance intersection [26]-[28] and exponential mixture density [29], [30] approach. However, the GA fusion has been observed suffering from a delay in detecting new targets [31], [32] and cardinality inconsistency [30] (e.g., underestimating the number of targets [33]), prone to missed detections [20], [25], [32], [34], [35] and vulnerable to non-overlapping fields of view [25], [36], [37]. In particular, the GA fusion may degrade [17], [25], [34], [35] with the increase of the number of fusing sensors and then does not suit large number sensor networks (LNSNs). 
In contrast to the GA fusion, attempts have also been made to apply the AA fusion in multisensor RFS filters since [38] which suggested multitarget likelihood averaging but gave no validation. It is, however, recently observed that the AA fusion (realized with appropriate mixture reduction or resampling operations) outperforms the GA fusion in the context of PHD fusion [17], [32], [37], [39]-[41], cardinalized PHD fusion [34], [35] and Bernoulli fusion [42], in addition to its much higher computing efficiency. The advantage of the AA fusion over the GA fusion has also been observed in the context of joint sensor localization and target tracking [43] and of non-RFS particle filtering [44]. Among these nontrivial comparisons are those from the research groups that make significant contribution on GA RFS fusion.

AA fusion complies with the law of "majority preference" (namely, minority is subordinate to majority) [17], [25], [32], [45] and is suitable for LNSNs. It is therefore interesting to see how the AA fusion rule should be extended for MB fusion and how it performs over the challenging LNSNs. Aiming exactly at the distributed, unknown-correlated LNSNs, the main contribution of this work is as follows

1) We prove that both GA and AA fusion rules are able to avoid double counting any information, but also dilute (count less than unit) the non-common information.

2) We point out that both $\mathrm{AA}$ and GA are Fréchet mean [46], [47], presenting more general analysis of the property of these two specific Fréchet means.

3) We propose the "target-wise fusion principle" to extend the AA fusion for fusing MBs, which divides the MB fusion problem into parallel Bernoulli-to-Bernoulli (B2B) fusion subproblems, each resolved by exact BernoulliAA fusion [42]. This result is significantly different from the existing MB-GA fusion [48]-[50] and also from existing AA fusion [17], [32], [34], [35], [37], [40].

4) We investigate both the average consensus [21], [22] and flooding [51] algorithms for internode communication, proposing accordingly two multidimensional assignment (MDA) approaches for B2B association based on either pairwise set matching or multi-sensor data clustering.

5) We extend the AA-fusion based cardinality consensus (CC) approach [39], [41] for MB fusion, proposing two communicatively and computationally efficient distributed MB filters, which merely disseminate and fuse information regarding target existence probabilities among local MB filters, but yield good results too.

This paper is organized as follows. Preliminaries are given in Section II. Properties of the AA and GA fusion are analyzed in Section III. The proposed B2B-AA-fusion-based distributed MB filters are presented in Section IV. Simulation results are given in Section V. We conclude in Section VI.

\section{PRELIMINARIES}

\section{A. RFS Modeling of MTDT}

The realization of an RFS of the multitarget states is a set $\mathbf{X}=\left\{\mathbf{x}_{1}, \ldots, \mathbf{x}_{n}\right\}$, where $n \geq 0$ is the number of targets which is random and $\mathbf{x}_{i} \in \mathbb{X} \subset \mathbb{R}^{d}$ is the state vector of the $i$-th target. The cardinality of an RFS $\mathbf{X}$ which indicates the number of elements is denoted with $|\mathbf{X}|$. The random nature of RFS $\mathbf{X}$ is captured by its probability density $\pi(\mathbf{X})$.

Target births follow an MB RFS independent of target survivals. Each target evolves and generates measurements independently. At time $k-1$, the target with state $\mathbf{x}_{k-1}$ will either die with probability $1-p_{k}^{\mathrm{s}}$ or persist at time $k$ with survival probability $p_{k}^{\mathrm{s}}$ and attains a new state $\mathbf{x}_{k}$ according to a Markov transition probability density function (PDF) $f_{k \mid k-1}\left(\mathbf{x}_{k} \mid \mathbf{x}_{k-1}\right)$.

Considering a sensor network composed of sensors $i=$ $1, \ldots, S$, we denote by $\mathcal{S}_{i}$ the set of neighbor sensors of sensor $i$. Given target with state $\mathbf{x}_{k}$, sensor $i$ either detects it with probability $p_{i, k}^{\mathrm{d}}$ and generates a measurement $\mathbf{z}_{i, k} \in \mathbf{Z}_{i, k}$ with likelihood $g_{i, k}\left(\mathbf{z}_{i, k} \mid \mathbf{x}_{k}\right)$ or fails to detect it with probability $1-p_{i, k}^{\mathrm{d}}$, where $\mathbf{Z}_{i, k}$ denotes the RFS of the measurements received at time $k$ by sensor $i$. The clutter follows a Poisson RFS, independent of target measurements.

\section{B. Cardinality and PHD}

Denote the set of all finite subsets of $\mathbb{X}$ is with $\mathcal{F}(\mathbb{X})$. For a function $f: \mathcal{F}(\mathbb{X}) \rightarrow \mathbb{R}$, the set integral in space $\chi$ is defined as [4, Ch. 11.3.3.1]

$\int_{\chi} f(\mathbf{X}) \delta \mathbf{X}=f(\emptyset)+\sum_{n=1}^{\infty} \frac{1}{n !} \int_{\chi^{n}} f\left(\left\{\mathbf{x}_{1}, \ldots, \mathbf{x}_{n}\right\}\right) d \mathbf{x}_{1} \ldots d \mathbf{x}_{n}$.

The cardinality distribution $\rho(n)$ of the RFS $\mathbf{X}$ is given as

$$
\begin{aligned}
\rho(n) & =\int_{|\mathbf{X}|=n} f(\mathbf{X}) \delta \mathbf{X} \\
& =\frac{1}{n !} \int_{\chi^{n}} f\left(\left\{\mathbf{x}_{1}, \ldots, \mathbf{x}_{n}\right\}\right) d \mathbf{x}_{1} \ldots d \mathbf{x}_{n} .
\end{aligned}
$$

The probability generating functional (PGFl) is an equivalent form of the multitarget process. By introducing a unitless test function $h: \mathbb{X} \rightarrow[0,1]$, the PGFl of RFS $\mathbf{X}$ is defined as

$$
G[h]=\int_{\chi} h^{\mathbf{X}} f(\mathbf{X}) \delta \mathbf{X},
$$

where the multitarget exponential notation is defined as $h^{\mathbf{X}}=$ $\prod_{\mathbf{x} \in \mathbf{X}} h(\mathbf{x})$ with $h^{\emptyset}=1$ and $0 \leq h(\mathbf{x}) \leq 1$.

The PHD $D(\mathbf{x})$ of the multitarget density is computed by taking the functional derivative of $G[h]$ in the direction of the Dirac delta density $\delta \mathbf{x}$ ( [4]) as follows

$$
D(\mathbf{x})=\left.\frac{\delta G}{\delta \mathbf{x}}[h]\right|_{h(\mathbf{x})=1} .
$$

\section{Bernoulli RFS}

The existence and non-existence of a single target can be modelled by a Bernoulli RFS. The cardinality distribution $\rho(n)$ of Bernoulli RFS $\mathbf{X}$ is Bernoulli, for which $\mathbf{X}$ can either be empty (with probability $1-r$ ) or have one element (with probability $r$ ), distributed over the state space according to PDF $p(\mathbf{x})$ [4]. That is, the FISST PDF of the Bernoulli RFS is given by

$$
f(\mathbf{X})= \begin{cases}1-r, & \text { if } \mathbf{X}=\emptyset \\ r p(\mathbf{x}), & \text { if } \mathbf{X}=\{\mathbf{x}\} \\ 0, & \text { otherwise }\end{cases}
$$


Lemma 1. Given $S$ Bernoulli RFSs with respective target existence probabilities $r_{i}$ and state PDFs $p_{i}(\mathbf{x}), i=1, \ldots, S$, and a nonnegative weight vector $W=\left\{w_{1}, \ldots, w_{S}\right\}$ subject to $\sum_{i=1}^{S} w_{i} r_{i} \leq 1$, their linear fusion is a Bernoulli RFS with target existence probability $\bar{r}$ and state PDF $\bar{p}(\mathbf{x})$ as follows

$$
\begin{aligned}
\bar{r} & =\sum_{i=1}^{S} w_{i} r_{i}, \\
\bar{p}(\mathbf{x}) & =\frac{1}{\sum_{i=1}^{S} w_{i} r_{i}} \sum_{i=1}^{S} w_{i} r_{i} p_{i}(\mathbf{x}) .
\end{aligned}
$$

Proof. This lemma is an extension of Lemma 1 of [42] by relaxing the constraint from $\sum_{i=1}^{S} w_{i}=1$ to $\sum_{i=1}^{S} w_{i} r_{i} \leq 1$. This relaxation does not matter the proof and so the proof given in [42] still holds.

\section{D. $M B R F S$}

An MB RFS $\mathbf{X}$ is the union of $M$ independent Bernoulli RFSs $\mathbf{X}^{(\ell)}$, namely, $\mathbf{X}=\bigcup_{\ell=1}^{M} \mathbf{X}^{(\ell)}$, which is completely characterized by a set of parameter pairs $\left\{\left(r^{(\ell)}, p^{(\ell)}(\mathbf{x})\right)\right\}_{\ell=1}^{M}$, where $r^{(\ell)}, p^{(\ell)}(\mathbf{x})$ denote the target existence probability and state density of the $\ell$-th $\mathrm{BC}$ or to say potential target $\mathbf{X}^{(\ell)}$, respectively.

The multitarget density of an MB RFS is given by

$$
\pi\left(\left\{\mathbf{x}_{1}, \ldots, \mathbf{x}_{n}\right\}\right)=\pi(\emptyset) \sum_{1 \leq i^{1} \neq \ldots \neq i^{n} \leq M} \prod_{j=1}^{n} \frac{r^{\left(i^{j}\right)} p^{\left(i^{j}\right)}\left(\mathbf{x}_{j}\right)}{1-r^{\left(i^{j}\right)}},
$$

where $n=\{1, \cdots, M\}$ and $\pi(\emptyset)=\prod_{j=1}^{M}\left(1-r^{\left(i^{j}\right)}\right)$.

The PGFl of the above MB RFS is [4, pp.660]

$$
G[h]=\prod_{\ell=1}^{M}\left(1-r^{(\ell)}+r^{(\ell)}\left\langle p^{(\ell)}, h\right\rangle\right),
$$

from which the PHD $D(\mathbf{x})$ can be calculated according to (3), yielding

$$
D(\mathbf{x})=\sum_{\ell=1}^{M} r^{(\ell)} p^{(\ell)}(\mathbf{x}) .
$$

\section{E. Fréchet-p-mean}

The Fréchet mean is a statistic used to characterize the central tendency of a distribution in arbitrary metric spaces [46], [47]. For a metric space $(\digamma, d(\cdot, \cdot))$, the Fréchet $p$-mean $\mu$ of a probability distribution $f$ is defined by

$$
\mu^{p}(f) \triangleq \underset{\nu \in \digamma}{\arg \min } \int_{\digamma} d^{p}(\nu, x) f(x) d x,
$$

where $d^{p}(\nu, x)$ is a given metric on the distance between $\nu$ and $x$ at the power $p$ and the content to be minimized, namely $\int_{\digamma} d^{p}(\nu, x) f(x) d x$, is called the Fréchet function.

The discrete analogue, the Fréchet $p$-mean of a data set $Z=\left\{z_{1}, \ldots, z_{S}\right\}$ with respect to a nonnegative weight vector $W=\left\{w_{1}, \ldots, w_{S}\right\}$, is defined by

$$
\mu^{p}(Z, W) \triangleq \underset{\nu \in \digamma}{\arg \min } \sum_{i=1}^{S} w_{i} d^{p}\left(\nu, z_{i}\right) .
$$

Clearly, the existence, possible uniqueness and values of the Fréchet $p$-mean depend on the metric $d(\cdot, \cdot)$. For real numbers, the AA is a Fréchet mean $(p=2)$ using the Euclidean distance as the metric (i.e., the Fréchet function gives the mean square error), while for positive real numbers, the GA is a Fréchet mean $(p=2)$ using the following distance function

$$
d^{2}(x, \nu)=\|\log (x)-\log (\nu)\|^{2} .
$$

\section{Properties And Reasoning for AA ANd GA}

For fusing probability distributions $f_{i}(\mathbf{X})$ and fusing weights $w_{i} \geq 0, i=1, \ldots, S$, subject to $\sum_{i=1}^{S} w_{i}=1$, the AA and GA are respectively given by

$$
\begin{aligned}
& f_{\mathrm{AA}}(\mathbf{X}) \triangleq \sum_{i=1}^{S} w_{i} f_{i}(\mathbf{X}), \\
& f_{\mathrm{GA}}(\mathbf{X}) \triangleq C^{-1} \prod_{i=1}^{S}\left(f_{i}(\mathbf{X})\right)^{w_{i}},
\end{aligned}
$$

where $C=\int \prod_{i=1}^{S}\left(f_{i}(\mathbf{X})\right)^{w_{i}} \delta \mathbf{X}$ is the normalized constant.

\section{A. Conservativeness}

Lemma 2. Both $A A$ and GA avoid double counting any information in the fusing sources

Proof. Double counting if any can only occur to the common information that is owned by multiple fusing sources. This is simply because any non-common information that does not belong to the other fusing sources will be counted less than unit in whether multiplication or power-calculation as long as the fusing weights are smaller than one. On one hand, if a fusion rule can avoid double counting in the extreme case of identical fusing sources in which the information of all fusing sources are identical, it must be able to avoid double counting in any other cases. This is simply because compared to the identical case, any other cases can be equally taken as that a part of the common information has been substituted by noncommon information in different fusing sources. Simply, this substitution will not cause double counting any information. On the other hand, in the identical fusion source case, the AA and GA become the same, identical to that of the fusing sources for which there is no information double counting. Combining these two sides, the lemma is proved.

More insights about the conservativeness of both fusion algorithms can be found in [20]. From the above reasoning, it can be seen that both average fusion rules deal with the unknown cross-correlation in an "extremely conservative" means (as they are able to resolve the worst case: all information are common). This is, however, more or less over conservative when there are non-common information which will be diluted in the convex fusing, i.e., counted less than unit.

\section{B. Fréchet Function (Fusion Divergence) Minimization}

As long as we can extend the Euclidean distance and the distance as defined in (11) from real numbers $x \in \mathbb{R}$ to realvalued functions $f: \mathcal{F}(\mathbb{X}) \rightarrow \mathbb{R}$, it can seen that both the AA 
as in (12) and the GA as in (13) are Fréchet $p$-mean. To be more specific, we have

$$
\begin{aligned}
& f_{\mathrm{AA}}(\mathbf{X})=\underset{g(\mathbf{X})}{\arg \min } \sum_{i=1}^{S} w_{i}\left\|f_{i}(\mathbf{X})-g(\mathbf{X})\right\|^{2}, \\
& f_{\mathrm{GA}}(\mathbf{X})=\underset{g(\mathbf{X})}{\arg \min } \sum_{i=1}^{S} w_{i}\left\|\log f_{i}(\mathbf{X})-\log g(\mathbf{X})\right\|^{2} .
\end{aligned}
$$

Indeed, $L^{2}$-distance has been used as a measure of the quality of the consensus [41], [42], [52], i.e., how much the consensus has been reached.

To note, the Fréchet function corresponding to a specific Fréchet mean may not be unique. Relevantly, as pointed out in [25], [53] and more formally w.r.t. RFS in [35], [45], the AA and GA fusion rules symmetrically minimizes the weighted sum of the directed Kullback-Leibler divergences between the fusing densities and the fused result, i.e.,

$$
\begin{aligned}
& f_{\mathrm{AA}}(\mathbf{X})=\underset{g(\mathbf{X})}{\arg \min } \sum_{i=1}^{S} w_{i} D_{\mathrm{KL}}\left(f_{i}(\mathbf{X}) \| g(\mathbf{X})\right), \\
& f_{\mathrm{GA}}(\mathbf{X})=\underset{g(\mathbf{X})}{\arg \min } \sum_{i=1}^{S} w_{i} D_{\mathrm{KL}}\left(g(\mathbf{X}) \| f_{i}(\mathbf{X})\right) .
\end{aligned}
$$

The Fréchet function minimization property indicates that all fusing sources make the last change to reach an agreement [43], which, however, is irrelevant with the the filter accuracy. A consensus is required or is just the goal for fusion in some cases like "aggregation" [53], [54] but not necessary the case for multisensor filtering. The reasoning or motivation for consensus or for aggregation cannot be taken as granted in the context of distributed filtering.

In addition to the popular AA and GA, there are various Fréchet means such as the known Harmonic mean, power mean and quasi-AA [47] as well as Kendall/Footrule optimal aggregation [54]. In fact, one can easily define a Fréchet mean. Let us say, for a derivable metric $\epsilon\left(f, f_{i}\right)$ between probability measures $f$ and $f_{i}$ in the metric space $(\digamma, \epsilon(\cdot, \cdot))$, suppose that its functional derivative with regard to $f$ is

$$
\Phi\left(f, f_{i}\right)=\frac{\delta \epsilon\left(f, f_{i}\right)}{\delta f},
$$

and setting $\sum_{i=1}^{S} w_{i} \Phi\left(f^{*}, f_{i}\right)=0$ yields

$$
f^{*}=\Psi\left(\left\{f_{i}\right\}_{i=1}^{S} ;\left\{w_{i}\right\}_{i=1}^{S}\right) .
$$

We may refer to such a metric $\epsilon\left(f, f_{i}\right)$ as simple metric. Clearly, (19) provides a fusion of $\left\{f_{i}\right\}_{i=1}^{S}$ using fusion weights $\left\{w_{i}\right\}_{i=1}^{S}$, which yields a Fréchet mean, i.e.,

$$
f^{*}=\underset{f \in \digamma}{\arg \min } \sum_{i=1}^{S} w_{i} \epsilon\left(f, f_{i}\right) .
$$

\section{Empirical Reasoning for AA fusion}

As addressed so far, the conservativeness and Fréchet mean properties of the AA fusion cannot explain why we need a particular average fusion rule like AA. More insights about this simple, linear fusion rule are needed.
1) Accuracy in time series: The AA fusion, carried out in the means of aggregating information and re-weighting them, preserves all information obtained by local fusing sensors. As a result, misdetection is greatly avoided at the price of increased risk for causing false alarms. To address the false alarm risk, the partial consensus approach [17], [32] that carries out fusion only on partial information which are more likely of targets and therefore reduces the involvement of false alarms is proven practically useful. More importantly, in the time series view, false alarms that by accident pass such an information censoring will easily be terminated in the subsequent filtering iterations. Furthermore, an additional step that is usually taken with the density-AA fusion is mixture reduction such as mixture merging and pruning [32], [39], which reduces the size of the mixture. When target state-estimates are extracted from the modes/peaks of the components, merging the components that likely represent the same target will get a more accurate mode/peak in general. However, we note that as proved in the Appendix of this paper, the standard Gaussian merging scheme [8], [55] does not change the mean and variance of the underlying distribution. Therefore, more benefit can be expected from advanced merging schemes that properly reduce the variance, e.g., [32]. There is still much space for further investigation in this regard.

2) Robustness to local errors/faults: Faults/errors in data such as misdetection, failure, clutter are common and form the key challenge to real MTDT applications. One of the key goals of multi-sensor collaboration is just to solve or compensate for the local sensor errors/faults. For this purpose, the AA fusion that follows a "majority preference" (i.e., minority is subordinate to majority), has obvious advantages in identifying and resolving local random errors; see the argument given in [32, Sec.III.B], [17, Sec.4] and also [25]. Further, recall that the AA fusion is more or less over-conservative unless the fusing densities are fully identical as explained in Section III-A. This "over-conservativeness" actually adds to the robustness of the filter to deal with model mismatching and disturbance when the estimator can easily be unbiased. These properties become increasingly prominent with the increase of the number of fusing sensors involved for which the risks of various fault/error increase too and for which many fusion approaches including the GA fusion may just degrade; see the experimental studies given in [17], [25], [34], [35].

3) Online fusion: Most RFS filters require either Gaussian mixture (GM) or particle filters for approximate calculation. As the result, the posterior is represented by either Gaussian components (GCs) or particles. The AA fusion admits exact calculation for either GM or particle implementation and is insensitive to the applying order of the sensors. In fact, as shown in (12), the AA fusion imposes mainly re-weighting calculation which is computationally very efficient. This is an important advantage since the filters cannot afford much extra time for internode communication and fusion calculation in real-time distributed tracking applications, unless they can be performed in parallel to the filtering calculation [17], [18].

The above properties and advantages render the AA fusion attractive and promising to the challenging LNSNs. 


\section{Proposed Target-Wise AA-MB Fusion}

At each sensor $i=1, \ldots, S$, the local MB filter recursively propagates and updates over time the MB posterior $\pi_{i}(\mathbf{X})$ which factories into BCs $\left\{\left(r_{i, k}^{(\ell)}, p_{i, k}^{(\ell)}(\mathbf{x})\right)\right\}_{\ell=1}^{M_{i, k}}$. For the detail of the classic single sensor $\mathrm{MB}$ filter, the reader is kindly referred to [10]. We hereafter address the proposed MBAA fusion algorithm based on the GM implementation. The approach can be extended to the particle implementation.

Given fusing MB densities $\pi_{i}(\mathbf{X})$ and fusing weights $w_{i} \geq$ $0, i \in \mathcal{S} \subseteq\{1, \ldots, S\}$, subject to $\sum_{i \in \mathcal{S}} w_{i}=1$, their straightforward AA fusion is given by

$$
\bar{\pi}(\mathbf{X})=\sum_{i \in \mathcal{S}} w_{i} \pi_{i}(\mathbf{X})
$$

This, however, is problematic in two aspects:

1) the result is no longer a MB density [45] and will therefore undermine the necessary MB-recursion if used.

2) this fusion disregards the individual $\mathrm{BC}$ information contained in the MB process, which is an advantageous feature of the MB filter as compared with the PHD filter.

Instead, we adopt a "divide and conquer" strategy, i.e., the target-like BCs in each sensor are associated with those in the neighbor sensors representing the same target (namely MDA for B2B association) to allow closed-form Bernoulli-AA fusion as addressed in Lemma 1, while the BCs that are more like false alarms will be excluded from the fusion. This choice is appropriate as it complies with the herein proposed "targetwise fusion principle": Fusion should be carried out with respect to the information of the same target and excludes false alarms. This is simply because it lacks physical significance to fuse the information of different targets or between targets and false alarms.

\section{A. MB Factorization and Partial Consensus}

Considering the GM implementation of the MB posterior, each BC $\left(r_{i, k}^{(\ell)}, p_{i, k}^{(\ell)}(\mathbf{x})\right)$ of sensor $i$ at time $k$ is represented by $J_{i, k}^{(\ell)}$ GCs weighted by $\omega_{i, k}^{(\ell, j)} \geq 0, j=1, \ldots, J_{i, k}^{(\ell)}$, i.e.,

$$
p_{i, k}^{(\ell)}(\mathbf{x}) \approx \sum_{j=1}^{J_{i, k}^{(\ell)}} \omega_{i, k}^{(\ell, j)} \mathcal{N}\left(\mathbf{x} ; \boldsymbol{\mu}_{i, k}^{(\ell, j)}, \boldsymbol{\Sigma}_{i, k}^{(\ell, j)}\right),
$$

where $\mathcal{N}(\mathbf{x} ; \boldsymbol{\mu}, \boldsymbol{\Sigma})$ denotes a Gaussian PDF with mean vector $\boldsymbol{\mu}$ and covariance matrix $\boldsymbol{\Sigma}$ and $\sum_{j=1}^{J_{i, k}^{(\ell)}} \omega_{i, k}^{(\ell, j)}=1$.

Clearly, the distribution of each BC $p_{i, k}^{(\ell)}(\mathbf{x})$ is determined by the parameter set $\mathcal{G}_{i, k}^{(\ell)} \triangleq\left\{\left(\omega_{i, k}^{(\ell, j)}, \boldsymbol{\mu}_{i, k}^{(\ell, j)}, \boldsymbol{\Sigma}_{i, k}^{(\ell, j)}\right)\right\}_{j=1, \ldots, J_{i, k}^{(\ell)}}$ from which we have

- The PHD is approximated as a set of GMs as follows

$$
D_{i}(\mathbf{x}) \approx \sum_{\ell=1}^{M_{i, k}} r_{i, k}^{(\ell)} \sum_{j=1}^{J_{i, k}^{(\ell)}} \omega_{i, k}^{(\ell, j)} \mathcal{N}\left(\mathbf{x} ; \boldsymbol{\mu}_{i, k}^{(\ell, j)}, \boldsymbol{\Sigma}_{i, k}^{(\ell, j)}\right) .
$$

- The Euclidean-mean-state of the $\ell$-th BC as expressed in (22) is calculated as follows

$$
\overline{\boldsymbol{\mu}}_{i, k}^{(\ell)}=\sum_{j=1}^{J_{i, k}^{(\ell)}} \omega_{i, k}^{(\ell, j)} \boldsymbol{\mu}_{i, k}^{(\ell, j)} .
$$

- By integrating the PHD, the number of targets, namely the cardinality of the MB RFS, is estimated as

$$
\hat{N}_{i, k}=\sum_{\ell=1}^{M_{i, k}} r_{i, k}^{(\ell)}
$$

Abiding by the partial consensus approach [32], only targetlike $\mathrm{BCs}$ (hereafter referred to as $T-B C s$ ) are disseminated and get involved in the B2B fusion while the false-alarm-like BCs $(F A-B C s)$ do not change ${ }^{1}$. This has also been proved very necessary for PHD-AA fusion [17], [32], [37].

Following the suggestion given in [32, Sec.III.A], we adopt two alternative rules to determine the T-BCs:

- Rank rule: Specify the number of T-BCs as equal to the estimated number of targets at each sensor, or slightly larger, and then take those with the largest target existence probabilities as the T-BCs.

- Threshold rule: Specify a threshold $r_{\text {gate }}$, and then only the BCs with target existence probability greater than that threshold are identified as the T-BCs ${ }^{2}$.

Without loss of generality, assume that BCs $\ell=1, \ldots, m_{i, k}$ at sensor $i$ are T-BCs while the rest $\ell=m_{i, k}+1, \ldots, M_{i, k}$ are FA-BCs. In what follows, we will address the B2B fusion in detail based on the consensus [21], [22] and flooding [51] schemes, respectively. Both are distributed algorithms in which all local sensors perform similar peer-to-peer (P2P) communication and fusion operations, in parallel. They have different communication/computation costs and convergence rates [17], [32], [42], and necessitate different B2B association methods as to be addressed below. In either case, the distance between two BCs is given by the $L^{2}$ distance between their mean states $\overline{\boldsymbol{\mu}}_{i, k}^{(\ell)}$ as calculated in (24) or between the states of the respective greatest weighted GCs from the two BCs. After B2B association, B2B fusion is performed for which we present three propositions that inherits from Lemma 1 and therefore need no proofs.

The procedures of both algorithms as to be detailed in the next two subsections are summarized in Algorithms 1 and 2, respectively. The distributed $\mathrm{MB}$ filters are referred to as $B 2 B$ $A A$-Flooding or $B 2 B-A A$-Consensus depending on whether the flooding or consensus algorithm is used. To note, it is not only the Output T-BCs of the fusion algorithm but also the unfused local FA-BCs $\left\{r_{i, k}^{(\ell)}, \mathcal{G}_{i, k}^{(\ell)}\right\}_{\ell=m_{i, k}+1}^{M_{i, k}}$ that will be used in the next filtering iteration $k+1$.

\section{B. MB B2B-Association and Fusion Based on Flooding}

We first consider the distributed flooding scheme [51]. In such a protocol, each local sensor equivalently serves as a fusion center which collects the relevant information from the other sensors via one or multiple $\mathrm{P} 2 \mathrm{P}$ communication iterations. Fusion only occurs at the end of the communication, once in each filtering iteration.

\footnotetext{
${ }^{1}$ One may consider abandoning these FA-BCs, which, however, may cause significant problem when any of the FA-BCs turn out to be real BCs. According to our experimental studies, the result will almost surely become worse if FA-BCs are abandoned.

${ }^{2}$ Setting $r_{\text {gate }}=0$ amounts to not applying partial consensus. However, as we found, the fusion result will degrade significantly if $r_{\text {gate }}$ is too small (e.g., $<0.1$ ). This indicates that the partial consensus approach is necessary.
} 


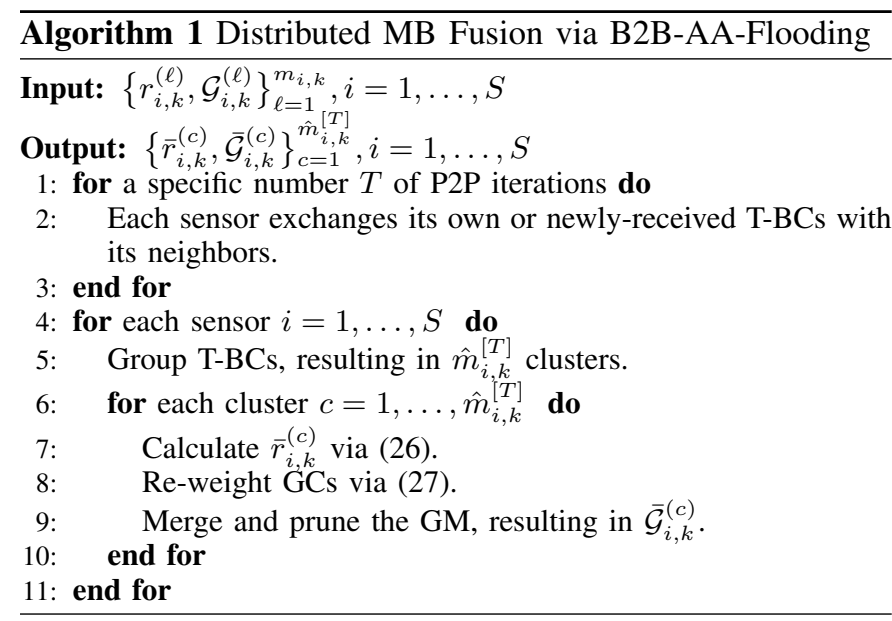

1) BCs flooding and clustering: At communication iteration $t=1, \ldots, T$, each sensor forwards all the T-BCs that have never been sent before to its neighbors and receives theirs. Let $\mathcal{S}_{i}^{[t]}$ denote the set of sensors that are at most $t$ hops away from sensor $i$, including sensor $i$ itself. Once flooding is completed at iteration $T$, sensor $i$ receives the parameter set $\left\{\left\{r_{v, k}^{(\ell)}, \mathcal{G}_{v, k}^{(\ell)}\right\}_{\ell=1}^{m_{v, k}}\right\}_{v \in \mathcal{S}_{i}^{[T]}}$. Then, the BCs are grouped via $\left\{\left\{\overline{\boldsymbol{\mu}}_{v, k}^{(\ell)}\right\}_{\ell=1}^{m_{v, k}}\right\}_{v \in \mathcal{S}_{i}^{[T]}}$ under two constraints:

- BCs from the same sensor should not be in the same cluster because each target forms no more than one BC at each sensor;

- the number of BCs in each cluster (namely the cluster size) should be larger than $\eta_{\mathrm{d}}\left|\mathcal{S}_{i}^{[T]}\right|$, where $0<\eta_{\mathrm{d}}<1$ is a factor to account for misdetection.

To this end, the constrained clustering method [56], [57] is readily available; for simplicity, the first constraint may not be strictly obeyed. We omit the detail here ${ }^{3}$.

Let us say, $\hat{m}_{i, k}^{[T]}$ clusters are finally formed and there are totally $J_{i, k}^{(c)}$ BCs in cluster $c=1, \ldots, \hat{m}_{i, k}^{[T]}$ formed at sensor $i$ at time $k$. Denote by $S_{i, k}^{(c)} \subseteq \mathcal{S}_{i}^{[T]}$ the set of the sensors that contribute one $\mathrm{BC}$ to cluster $c$. We will now use the same superscript $c$ to refer to all the relevant parameters belong to cluster $c$, i.e., $\forall v \in S_{i, k}^{(c)}, j=1, \ldots, J_{i, k}^{(c)}$, $\left(r_{v, k}^{(\ell)}, \omega_{v, k}^{(\ell, j)}, \boldsymbol{\mu}_{v, k}^{(\ell, j)}, \boldsymbol{\Sigma}_{v, k}^{(\ell, j)}\right)$ that are grouped to cluster $c$ will be re-denoted as $\left(r_{v, k}^{(c)}, \omega_{v, k}^{(c, j)}, \boldsymbol{\mu}_{v, k}^{(c, j)}, \boldsymbol{\Sigma}_{v, k}^{(c, j)}\right)$, respectively. Then, within each cluster AA-Bernoulli fusion is carried out.

2) $B 2 B A A$ fusion within clusters: To realize Lemma 1 by treating all sensors equally (i.e., using uniform fusion weights), a new $\mathrm{BC}$ is generated in cluster $c=1, \ldots, \hat{m}_{i, k}^{[T]}$ as follows,

Proposition 1. The mean target existence probability is given as follows

$$
\bar{r}_{i, k}^{(c)}=\frac{1}{\left|S_{i, k}^{(c)}\right|} \sum_{v \in S_{i, k}^{(c)}} r_{v, k}^{(c)}
$$

\footnotetext{
${ }^{3}$ The Matlab codes of the flooding-and-then-clustering algorithm can be found in the URL: sites.google.com/site/tianchengli85/matlab-codes/c4f, for which the corresponding simulation setup was given in [56].
}

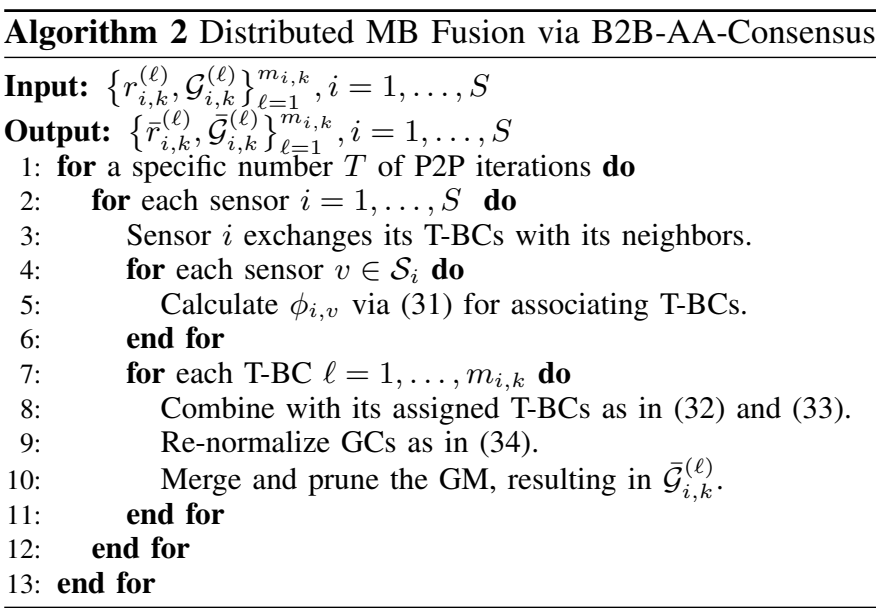

Further, all the GCs in cluster $c$ of sensor $i$ will be renormalized (without changing their means and covariances) as follows, $\forall v \in S_{i, k}^{(c)}, j=1, \ldots, J_{v, k}^{(c)}$,

$$
\bar{\omega}_{v, k}^{(c, j)}=C^{-1} r_{v, k}^{(c)} \omega_{v, k}^{(c, j)},
$$

with the normalization factor $C=\sum_{v \in S_{i, k}^{(c)}} \sum_{j=1}^{J_{v, k}^{(c)}} r_{v, k}^{(c)} \omega_{v, k}^{(c, j)}$.

The above local GM set union and reweighting operations result in an arithmetically averaged BC state density

$$
\bar{p}_{i, k}^{(c)}(\mathbf{x})=\frac{1}{\sum_{v \in \mathcal{S}_{i}^{[T]}} r_{v, k}^{(c)}} \sum_{v \in \mathcal{S}_{i}^{[T]}} r_{v, k}^{(c)} p_{v, k}^{(c)}(\mathbf{x}) .
$$

This, together with (26), constitute the AA-Bernoulli fusion [42, Sec.III]. After this, GM merging, capping and pruning [8, Sec.III.C] are applied to each cluster for reducing the number of GCs, which result in the final AA-fused GM parameters, denoted as $\overline{\mathcal{G}}_{i, k}^{(c)} \triangleq\left\{\left(\bar{\omega}_{i, k}^{(c, j)}, \boldsymbol{\mu}_{i, k}^{(c, j)}, \boldsymbol{\Sigma}_{i, k}^{(c, j)}\right)\right\}_{j=1, \ldots, \tilde{J}_{i, k}^{(c)}}$.

Finally, to ensure the sum of the existence probabilities of all BCs at sensor $i$ equal to the mean cardinality, namely floodingbased CC [32], [41], given by

$$
\begin{aligned}
\hat{\bar{N}}_{i, k} & =\frac{1}{\left|\mathcal{S}_{i}^{[T]}\right|} \sum_{v \in \mathcal{S}_{i}^{[T]}} \hat{N}_{v, k} \\
& =\frac{1}{\left|\mathcal{S}_{i}^{[T]}\right|} \sum_{v \in \mathcal{S}_{i}^{[T]}} \sum_{\ell=1}^{M_{v, k}} r_{v, k}^{(\ell)},
\end{aligned}
$$

normalization is needed to be applied to both $\left\{\bar{r}_{i, k}^{(c)}\right\}_{c=1}^{\hat{m}_{i, k}^{[T]}}$ and $\left\{r_{i, k}^{(\ell)}\right\}_{\ell=m_{i, k}+1}^{M_{i, k}}$ by multiplying the following factor

$$
\alpha_{i, k} \triangleq \frac{\hat{\bar{N}}_{i, k}}{\sum_{c=1}^{\hat{m}_{i, k}^{[T]}} \bar{r}_{i, k}^{(c)}+\sum_{\ell=m_{i, k}+1}^{M_{i, k}} r_{i, k}^{(\ell)}} .
$$

\section{MB B2B-Association and Fusion Based on Consensus}

This section addresses the consensus algorithm [21], [22] for internode $\mathrm{P} 2 \mathrm{P}$ communication. Here, $\mathrm{BCs}$ of neighbor sensors need to be associated, which is a NP-hard MDA problem when more than two sensors are involved. To solve this problem, we decompose the MDA problem into a sequence of $2 \mathrm{D} /$ pairwise 
B2B association sub-problems, each resolved by the Munkres' method [58] (a.k.a. the Hungary algorithm) in polynomial time. We first mentioned such an idea in [45].

1) B2B association: To associate the T-BCs from sensor $i$ and its neighbor $v \in \mathcal{S}_{i}$, a $m_{i, k} \times m_{v, k}$ distance matrix $D_{i, v}$ (if $m_{i, k} \leq m_{v, k}$ otherwise transpose the matrix) is constructed with element $d_{\ell, \ell^{\prime}} \triangleq\left\|\overline{\boldsymbol{\mu}}_{i, k}^{(\ell)}-\overline{\boldsymbol{\mu}}_{v, k}^{\left(\ell^{\prime}\right)}\right\|_{2}$, where $\ell=1, \ldots, m_{i, k}$, $\ell^{\prime}=1, \ldots, m_{v, k}$. The Munkres' assignment results in the optimal assignment $\left(\ell, \phi_{i, v}(\ell)\right), \ell=1, \ldots, m_{i, k}$ as follows

$$
\phi_{i, v}=\underset{\phi \in \Pi_{m_{v, k}}}{\operatorname{argmin}} \sum_{\ell=1}^{m_{i, k}} d_{\ell, \ell^{\prime}},
$$

where $\Pi_{m}$ denotes the set of all permutations on $\{1, \ldots, m\}$ for any positive integer $m$. For simplicity, we use the shorthand notation $\ell_{i}^{v}=\phi_{i, v}(\ell)$ and so $\ell_{i}^{i}=\ell$.

Before the fusion is carried out, an extra step is required to refine the assignments so that only the assigned pairs that are close enough to each other will be further considered; any assignment that does not meet the gating is considered as weak association and will be unassigned. These unassigned T-BCs, as same as the FA-BCs, will not be involved in fusion. Overall, this $\mathrm{BC}$ assignment procedure is analogous to what we have proposed for GC assignment [32, Sec.IV.C].

2) B2B Fusion: Let $\tilde{\mathcal{S}}_{i}^{(\ell)} \subseteq\left\{i, \mathcal{S}_{i}\right\}$ denote all the sensors that have one $\mathrm{T}-\mathrm{BC}$ match and be assigned with $\mathrm{BC} \ell$ at sensor $i$. For each $\ell=1, \ldots, m_{i, k}$, the AA-Bernoulli fusion is given by the following proposition.

Proposition 2. Assigned BCs from neighbor sensors $v^{\prime} \in$ $\tilde{\mathcal{S}}_{i}^{(\ell)}$ are averaged to a single BC at sensor $i$ as follows

$$
\begin{gathered}
\bar{r}_{\tilde{\mathcal{S}}_{i}^{(\ell)}, k}^{(\ell)}=\frac{\sum_{v^{\prime} \in \tilde{\mathcal{S}}_{i}^{(\ell)}} w_{i, v^{\prime}} r_{v^{\prime}, k}^{\left(\ell_{i}^{v^{\prime}}\right)}}{\sum_{v^{\prime} \in \tilde{\mathcal{S}}_{i}^{(\ell)}} w_{i, v^{\prime}}}, \\
\bar{p}_{\tilde{\mathcal{S}}_{i}^{(\ell)}, k}^{(\ell)}=\frac{\sum_{v^{\prime} \in \tilde{\mathcal{S}}_{i}^{(\ell)}} w_{i, v^{\prime}} r_{v^{\prime}, k}^{\left(\ell_{i}^{v^{\prime}}\right)} p_{v^{\prime}, k}^{\left(\ell_{i}^{v^{\prime}}\right)}}{\sum_{v^{\prime} \in \tilde{\mathcal{S}}_{i}^{(\ell)}} w_{i, v^{\prime}} r_{v^{\prime}, k}^{\left(\ell^{v^{\prime}}\right)}},
\end{gathered}
$$

where the fusing weights $w_{i, v^{\prime}}$ are given by the Metropolis weights [21], [22] for fast convergence.

To implement (33), the relevant GCs from sensor $v^{\prime} \in \tilde{\mathcal{S}}_{i}^{(\ell)}$ need to be re-weighted and normalized (without changing their means and covariances), respectively, as follows

$$
\omega_{v^{\prime}, k}^{\left(\ell_{i}^{v^{\prime}}, j\right)}=C^{-1} w_{i, v^{\prime}} r_{v^{\prime}, k}^{\left(\ell_{i}^{v^{\prime}}\right)} \omega_{v^{\prime}, k}^{\left(\ell_{i}^{v^{\prime}}, j\right)}
$$

with $C=\sum_{v^{\prime} \in \mathcal{S}_{i}} \sum_{j=1}^{J_{v^{\prime}, k}^{\left(\ell^{v^{\prime}}\right)}} w_{i, v^{\prime}} r_{v^{\prime}, k}^{\left(\ell_{i}^{v^{\prime}}\right)} \omega_{v^{\prime}, k}^{\left(\ell_{i}^{v^{\prime}}, j\right)}$.

Clearly, if $\tilde{\mathcal{S}}_{i}^{(\ell)}=\left\{i, \mathcal{S}_{i}\right\}$, we have $\sum_{v^{\prime} \in \tilde{\mathcal{S}}_{i}^{(\ell)}} w_{i, v^{\prime}}=1$. This leads to the ideal case that, all BCs corresponding to the same target $\ell$ from different sensors in $\left\{i, \mathcal{S}_{i}\right\}$ are correctly assigned and fused in the AA fusion as follows

$$
\begin{aligned}
\bar{r}_{i, k}^{(\ell)} & =w_{i, i} r_{i, k}^{(\ell)}+\sum_{v \in \mathcal{S}_{i}} w_{i, v} r_{v, k}^{\left(\ell_{i}^{v}\right)}, \\
\bar{p}_{i, k}^{(\ell)} & =\frac{w_{i, i} r_{i, k}^{(\ell)} p_{i, k}^{(\ell)}+\sum_{v \in \mathcal{S}_{i}} w_{i, v} r_{v, k}^{\left(\ell_{i}^{v}\right)} p_{v, k}^{\left(\ell_{i}^{v}\right)}}{\bar{r}_{i, k}^{(\ell)}} .
\end{aligned}
$$

Otherwise, we only have $\bar{r}_{\tilde{\mathcal{S}}_{i}^{(\ell)}, k}^{(\ell)} \approx \bar{r}_{i, k}^{(\ell)}, \bar{p}_{\tilde{\mathcal{S}}_{i}^{(\ell)}, k}^{(\ell)} \approx \bar{p}_{i, k}^{(\ell)}$.
To reduce the size of the combined GM for each BC, GM merging and pruning may be applied. Furthermore, for $\mathrm{MB}$ RFS CC, the target existence probabilities may be finally rescaled as addressed in the following Proposition 3, which can be performed in parallel to the B2B fusion. With some notational abuse, we denote the final $M_{i, k}$ BCs (including both fused BCs and the BCs that do not take a part in fusion) at sensor $i$ at time $k$, with target existence probabilities $\bar{r}_{i, k}^{(\ell)}$ and GM parameters $\overline{\mathcal{G}}_{i, k}^{(\ell)}, \ell=1, \ldots, M_{i, k}$.

\section{Cardinality-only Consensus}

It is seen from Lemma 1 that, the fusion of $r_{i, k}^{(\ell)}$ is independent of $p_{i}(\mathbf{x})$. Therefore, we can disseminate $\left\{\left(r_{i, k}^{(\ell)}, \overline{\boldsymbol{\mu}}_{i, k}^{(\ell)}\right)\right\}_{\ell=1}^{m_{i, k}}$ and fuse $\left\{\left(r_{i, k}^{(\ell)}\right)\right\}_{\ell=1}^{m_{i, k}}$ merely. Then, only operations related to B2B association and fusing $\left\{\left(r_{i, k}^{(\ell)}\right)\right\}_{\ell=1}^{m_{i, k}}$ remain the same and operations related to fusing GM parameters are avoided. We refer to this approach as $B 2 B-A A$ $C C$. Although this scheme can be implemented via either flooding or the average consensus algorithm as addressed, we investigate the consensus approach only in our simulation, namely $B 2 B-A A-C C$-Consensus. To be more specific, in this approach, only $\mathrm{B} 2 \mathrm{~B}$ association operation and calculations as in (32) are required.

To further reduce the communication and to avoid the B2B association operation, one may only disseminate the overall estimate $\hat{N}_{i, k}$ of the MB RFS cardinality as given in (25), and average it with those from the neighbors sensors, leading to a protocol same to the standard $C C$ originally proposed for PHD fusion [41]. Then, only a single real value needs to be disseminated and fused per communication iteration. That is, starting from $\hat{N}_{i, k}^{[0]}=\hat{N}_{i, k}$, the following Proposition is applied, as a partial realization of Lemma 1 .

Proposition 3. In the consensus-based standard AA-CC approach, the following recursion calculation is carried out among each sensor $i$ and its neighbor sensors at communication iteration $t=1, \cdots, T$,

$$
\hat{N}_{i, k}^{[t]}=\sum_{v^{\prime} \in\left\{i, \mathcal{S}_{i}\right\}} w_{i, v^{\prime}} \hat{N}_{v^{\prime}, k}^{[t-1]} .
$$

At the end of communication iteration $T$ in each filtering iteration $k$, the result $\hat{N}_{i, k}^{[T]}$ is used to scale all target existence probabilities as follows, $\forall i=1, \ldots, M_{i, k}$,

$$
\bar{r}_{i, k}^{(\ell)}=\frac{r_{i, k}^{(\ell)}}{\sum_{\ell=1}^{M_{i, k}} r_{i, k}^{(\ell)}} \hat{N}_{i, k}^{[T]} .
$$

\section{E. Realization of $M D A$}

Convergence analysis and experimental comparison between the consensus and flooding algorithms are available in [51] and [17], [32], [41], [42]. However, in the context of B2B fusion based on MDA, they have new features due to the use of the clustering or 2D assignment algorithms. As shown next in our simulation, they lead to greatly different results.

The number of BCs remains the same in the consensusbased B2B fusion, i.e., each original BC will either be unchanged or be averaged with those received from the other 
sensors. No new BC is created. In contrast, the final number of BCs obtained in the flooding-based B2B-AA fusion depends on the clustering scheme and may be somehow different from the original number of BCs. The clustering algorithm can generate new BCs that are totally missed by a local sensor and therefore can better compensate for the locally missed detection as compared with the B2B-AA consensus algorithm. This capacity is important for MTDT.

Most clustering methods enjoy good performance for rich data but also suffers from the greatly increased computation due to the increased data size. As such, it can be expected that the flooding-based B2B-AA fusion which complies with data clustering is suitable for LNSNs, for which, however, the computation and communication cost will also be high.

\section{Simulation Results}

We considered the region of interest (ROI) given by $[-2 \mathrm{~km}, 2 \mathrm{~km}] \times[-2 \mathrm{~km}, 2 \mathrm{~km}]$. The target state is denoted as $\mathbf{x}_{k}=\left[\begin{array}{lllll}x_{k} & \dot{x}_{k} & y_{k} & \dot{y}_{k} & \omega_{k}\end{array}\right]^{\mathrm{T}}$ with planar position $\left[\begin{array}{ll}x_{k} & y_{k}\end{array}\right]^{\mathrm{T}}$, velocity $\left[\begin{array}{ll}\dot{x}_{k} & \dot{y}_{k}\end{array}\right]^{\mathrm{T}}$ and turn rate $\omega_{k}$. The number of objects is time varying due to random births and deaths. The target birth is an MB process described with parameters $\left\{r_{\mathrm{B}, k}^{(\ell)}, p_{\mathrm{B}, k}^{(\ell)}(\mathbf{x})\right\}_{\ell=1}^{4}$, where $r_{\mathrm{B}, k}^{(1)}=r_{\mathrm{B}, k}^{(2)}=0.02$, $r_{\mathrm{B}, k}^{(3)}=r_{\mathrm{B}, k}^{(4)}=0.03$, and $p_{\mathrm{B}, k}^{(\ell)}(\mathbf{x})=\mathcal{N}\left(\mathbf{x} ; \mu_{\mathrm{B}}^{(\ell)}, \boldsymbol{\Sigma}_{\mathrm{B}}\right)$ with $\mu_{\mathrm{B}}^{(1)}=[-1500,0,250,0,0]^{\mathrm{T}}, \mu_{\mathrm{B}}^{(2)}=[-250,0,1000,0,0]^{\mathrm{T}}$, $\mu_{\mathrm{B}}^{(3)}=[250,0,750,0,0]^{\mathrm{T}}, \quad \mu_{\mathrm{B}}^{(4)}=[1000,0,1500,0,0]^{\mathrm{T}}$, $\boldsymbol{\Sigma}_{\mathrm{B}}=\operatorname{diag}\left([50,50,50,50,6(\pi / 180)]^{\mathrm{T}}\right)^{2}$.

The probability of target survival is $P_{k}^{\mathrm{S}}=0.98$. The survival single-target movement follows a coordinated turn (CT) model with a sampling period of $1 s$ and transition density $f_{k \mid k-1}\left(\mathbf{x}_{k} \mid \mathbf{x}_{k-1}\right)=\mathcal{N}\left(\mathbf{x}_{k} ; F\left(\omega_{k}\right) \mathbf{x}_{k}, \mathbf{Q}\right)$, where

$$
F(\omega)=\left[\begin{array}{ccccc}
1 & \frac{\sin \omega}{\omega} & 0 & -\frac{1-\cos \omega}{\omega} & 0 \\
0 & \cos \omega & 0 & -\sin \omega & 0 \\
0 & \frac{1-\cos \omega}{\omega} & 1 & \frac{\sin \omega}{\omega} & 0 \\
0 & \sin \omega & 0 & \cos \omega & 0 \\
0 & 0 & 0 & 0 & 1
\end{array}\right],
$$

and $\mathbf{Q}=\operatorname{diag}\left(\left[\mathbf{G}, \mathbf{G}, \sigma_{u}^{2}\right]\right)$ with

$$
\mathbf{G}=\left[\begin{array}{cc}
\frac{\sigma_{w}^{2}}{4} & \frac{\sigma_{w}^{2}}{2} \\
\frac{\sigma_{w}^{2}}{2} & \sigma_{w}^{2}
\end{array}\right]
$$

and $\sigma_{w}=5 \mathrm{~m} / \mathrm{s}^{2}$, and $\sigma_{u}=(\pi / 180) \mathrm{rad} / \mathrm{s}$.

We considered the following observation model. Each sensor $i$ has a target detection probability 0.9 and a nonlinear range-bearing measurement model given as follows

$$
\mathbf{z}_{i, k}=\left[\begin{array}{c}
\sqrt{\left(x_{k}-x^{(i)}\right)^{2}+\left(y_{k}-y^{(i)}\right)^{2}} \\
\tan ^{-1}\left(\frac{x_{k}-x^{(i)}}{y_{k}-y^{(i)}}\right)
\end{array}\right]+\left[\begin{array}{c}
v_{i, k}^{(1)} \\
v_{i, k}^{(2)}
\end{array}\right],
$$

where $x^{(i)}$ and $y^{(i)}$ are the coordinates of sensor $i, v_{s, k}^{(1)}$ and $v_{i, k}^{(2)}$ are, individually, independent identical distributed zero-mean Gaussian with standard deviation $\sigma_{1}=10 \mathrm{~m}$ and $\sigma_{2}=(\pi / 90) \mathrm{rad}$, respectively. The field of view of the nonlinear sensors is a disk of radius $5 \mathrm{~km}$ centered at the sensor position; this disk always covers the entire ROI. The clutter measurements of different sensors are independent which uniformly distributed over each sensor's field of view with an average number of $r_{\mathrm{c}}$ clutter measurements per time step, or equivalently clutter intensity $\kappa_{k}\left(\mathbf{z}_{k}\right)=r_{\mathrm{c}} /(2 \pi \cdot 5000)$. We used $r_{\mathrm{c}}=5$ in our simulation.

We considered two scenarios. The first scenario is composed of 5 targets of deterministic trajectories (which were generated by the mentioned CT model without using process noises) and 50 sensors which are deployed and connected by 187 edges as shown in Fig. 1. The second scenario is composed of 10 targets whose trajectories were generated by the mentioned CT model using the random process noise $\mathbf{Q}$ and 15 sensors which form a network topology of grids as shown in Fig 6. The diameter of both networks is $D_{m}=6$. For both scenarios, we considered different numbers of communication iterations $T=1, \ldots, 6$ to evaluate the performance of the filters under different degrees of consensus. A larger $T$ indicates a higher degree of consensus. For the flooding scheme, the network achieves complete consensus when $T=D_{m}$ [51].

The local GM-MB filters employ the unscented approach to deal with the nonlinearity [10]. Each local filter uses at most 20 BCs and each BC comprises at most 10 GCs to keep the filter computationally efficient, prune the BCs with target existence probability lower than 0.01 , prune GCs with weights below 0.001 and merge those with Mahalanobis distance below 4 . The T-BCs are identified by using the threshold rule with threshold $r_{\text {gate }}=0.3$ as addressed in Section.IV.A. We use the factor $\eta_{\mathrm{d}}=0.5$ in the clustering algorithm with the B2BAA-Flooding approach. We reiterate that the partial consensus approach and the target-wise fusion principle are crucial for the AA fusion. Following the advice of [10, Sec.III.D], the number of targets is estimated from the posterior cardinality distribution by taking its mode and the target state is estimated from the individual modes from confirmed T-BC densities.

The proposed B2B-AA-Flooding, B2B-AA-Consensus, B2B$A A-C C$ and Standard $A A-C C$ were compared with the noncooperative approach that does not carry out any internode communication and fusion. At first, we considered the GAMB approach [49], [50] but it turned out that they (based on our implementation) are computationally intensive and do not work well with so many sensors as we consider here; indeed the GA fusion demonstrated degradation [17], [25], [34], [35] with the increase of the number of fusing sensors.

Filter performance is evaluated by the optimal subpattern assignment (OSPA) error with cut-off $c=2 \mathrm{~km}$ and order $\rho=2$ [59]. 50 simulation runs are performed with 100 time steps each run. Specifically, the average of the OSPA errors obtained by all the sensors is referred to as network OSPA error (in short $\mathrm{N}$-OSPA) and the average of the N-OSPAs over all the 100 time steps is referred to as time-averaged network OSPA error (TN-OSPA). We also consider the average communication cost (ACC) of the various filters by the number of real values broadcast by a sensor to its neighbors during all the dissemination iterations performed in one filtering iteration, averaged over all the sensors, time steps, and simulation runs. Note that each 1D $r_{i, k}^{(\ell)}$, 2D position components of $\overline{\boldsymbol{\mu}}_{v, k}^{(\ell)}$, 1D $\omega_{i, k}^{(\ell, j)}$, 5D $\boldsymbol{\mu}_{i, k}^{(\ell, j)}$, 5D symmetric matrix $\boldsymbol{\Sigma}_{i, k}^{(\ell, j)}$ take the 
communication of $1,2,1,5,15$ real values, respectively.

The results are shown for the two scenarios as follows: Fig. 2 and Fig. 7 show the averaged estimate of the number of targets and N-OSPA of different distributed MB filters and the non-cooperative $\mathrm{MB}$ filter, versus filtering time $k$. To better illustrate the result, target position estimates of the noncooperative filter and the $B 2 B-A A-F l o o d i n g$ (when $T=6$ ) filter at a single sensor are given in Fig. 1 and Fig. 6. Fig. 3 and Fig. 8 show the TN-OSPA of different filters over time. Fig. 4 and Fig. 9 show the ACC of different distributed MB filters versus the total number $T$ of communication iterations. Fig. 5 and Fig. 10 show the computing time for each filtering iteration of different distributed $\mathrm{MB}$ filters versus the total number $T$ of communication iterations.

In brief, the proposed four fusion approaches: Standard $A A$ $C C, B 2 B-A A-C C, B 2 B-A A-C o n s e n s u s$ and B2B-AA-Flooding improve the estimation accuracy of the number and states of the targets in an ascending order, while their costs in communication and computation are also ranked in an ascending order. We highlight the following findings:

- The performance of $B 2 B-A A-F l o o d i n g$ is significantly the best in all, although its cost in communication and computation is high and increases unsurprisingly fast with the increase of $T$. Both the strengths and weaknesses are due to the clustering algorithm; see our analysis given in Sec.IV-E. Outstanding performance of the clustering method for target detection has also been recently validated in [56], [60]. However, when $T=1$ (like "diffusion" [61], only direct neighbors exchange information with each other and no further communication occurs at each filtering iteration), the communication cost and computing time of the flooding based B2B approach are close to those of the consensus-based algorithm, but its OSPA reduction is much more significant. For further improvement, communication saving approaches and faster clustering are to be developed.

- Compared to $B 2 B-A A-C C$, the gain due to $B 2 B-A A$ Consensus is not so significant, although its communication and computation costs are much higher. $B 2 B-A A$ Consensus is composed of two types of operations for revising the $\mathrm{MB}$ posterior: 1) re-weight the BCs (referred to as "cardinality fusion"), 2) add new GCs to each T-BC and re-normalize all GCs within each fused $\mathrm{BC}$ ("PDF fusion"). $B 2 B-A A-C C$ has the same "cardinality fusion" operation but no "PDF fusion". In the current $B 2 B-A A$ Consensus implementation, the $\mathrm{B} 2 \mathrm{~B}-\mathrm{MDA}$ problem is resolved in an means of iterative pairwise B2B association. Disregarding the computational efficiency, accuracy improvement may be obtained by directly solving the MDA problem using approximate Lagrangian relaxation [62] or stochastic sampling approach [63] or by introducing a scheme to compensate for local missed detection.

- The standard AA-CC approach performs good in the first scenario considering that its communication and computation cost is extremely small. But its gain in the second scenario is smaller and is unstable (even degrading with the increase of the number of communication iterations from $T=2$ to $T=4$ ). There is probably because the

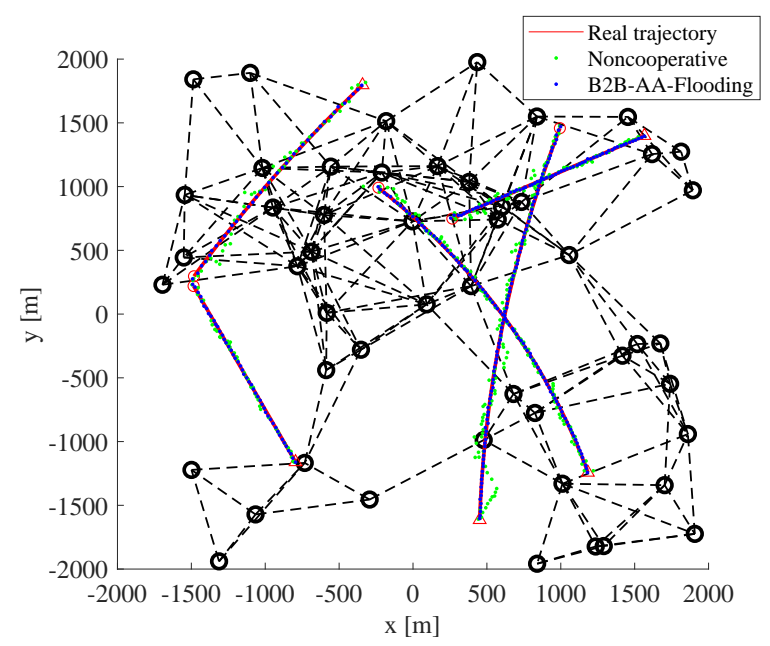

Fig. 1. ROI of the 1st simulation scenario consisting of 50 sensors (positioned at black circles) connected by 187 communication links (given by black dashed lines) and 5 targets of smooth trajectories (red lines). Green and blue points are estimates of the target positions at different times in a single run achieved by the non-cooperative and B2B-AA-flooding (when $T=6$ ) filters, respectively, at the same sensor.

number of targets is underestimated somehow in time interval $k \in[40,100] \mathrm{s}$ by almost all filters (as shown in the upper sub-figure of Fig. 7), i.e., missed detection is frequent. In such a case, the accuracy gain of the AA fusion cannot be guaranteed. Further, we note that in our implementation the number of targets is estimated from the posterior cardinality distribution by taking its mode, not its mean and so the original estimate of the number of targets is not unbiased.

- In almost all distributed algorithms, both the gain in reducing the OSPA and the communication cost increase slower while their computation time costs increase linearly with the increase of $T$, after $T=2$. The reduction of the OSPA is the most significant from $T=0$ to $T=1$. That means, more iterations of communication and fusion do not benefit the filter much after $T=2$. This is consistent to the findings shown in the AAPHD/Bernoulli fusion [17], [32], [41], [42].

\section{CONCLUSiON}

While the arithmetic average (AA) fusion is simple, fundamental and widely used in many fields, it is only in the last several years that its performance has been investigated in-depth in the context of RFS fusion. We point out that it, as well as the geometric average, is a Fréchet mean. New insights and discussion are provided based on a comprehensive literature study in this paper. A significant extension of the cutting edge AA-RFS fusion is made for accommodating the MB filter based on the target-wise fusion principle. Implementation details are provided for four various AA-fusion based distributed MB filters that have different computation and communicative costs. Simulations have demonstrated their effectiveness, computing efficiency and accuracy performance under a series of numbers of communication iterations in two different scenarios. It is our future work to extend the AA fusion and the proposed B2B association algorithms to the labelled RFS fusion framework [64], [65]. 

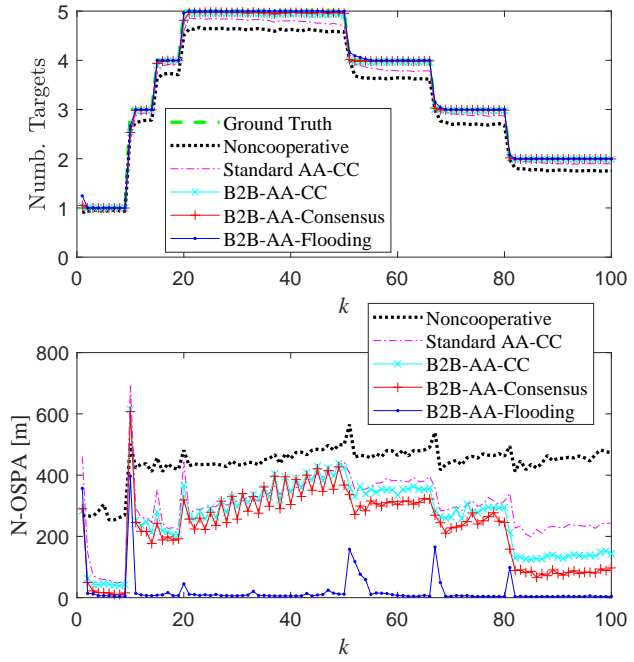

Fig. 2. Average estimated number of targets and N-OSPA of different filters over time in the 1 st scenario.

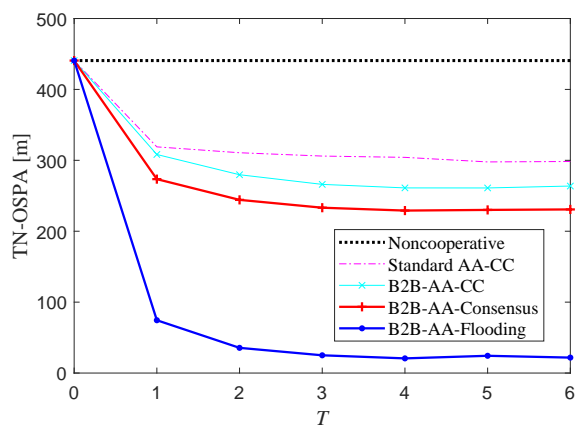

Fig. 3. TN-OSPAs of different filters over time for different numbers $T$ of communication iterations in the 1 st scenario.

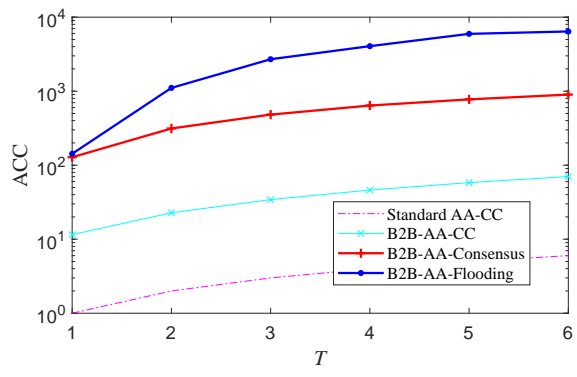

Fig. 4. ACCs of different filters for different numbers $T$ of communication iterations in the 1 st scenario.

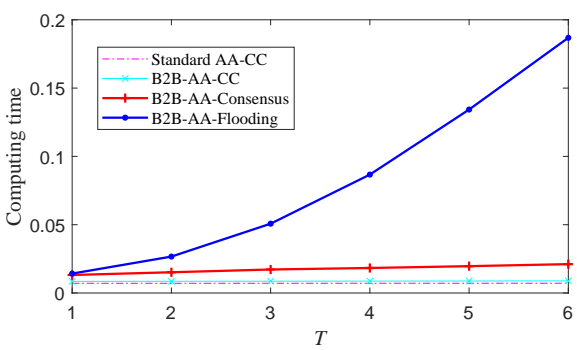

Fig. 5. Computing time for each filtering iteration of different filters for different numbers $T$ of communication iterations in the 1st scenario.

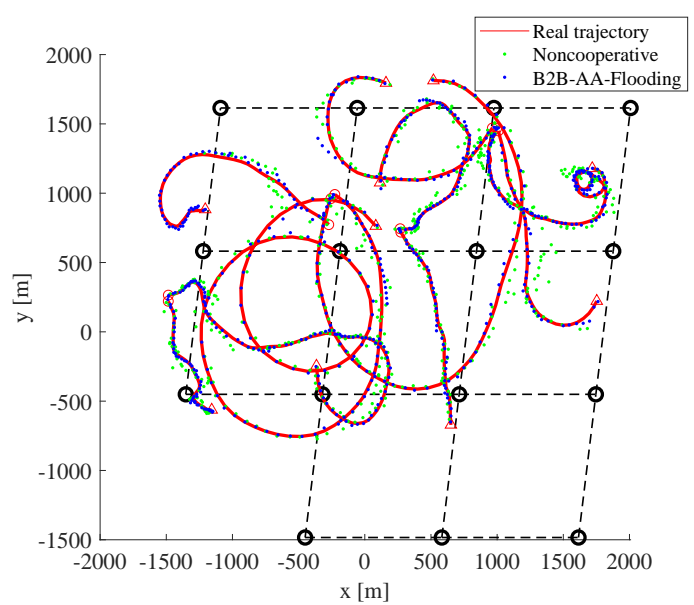

Fig. 6. ROI of the 2 nd scenario consisting of 15 sensors connected by 22 communication links and 10 targets with various trajectories. Green and blue points are estimates of the target positions at different times in a single run achieved by the non-cooperative and B2B-AA-flooding (when $T=6$ ) filters, respectively, at the same sensor.
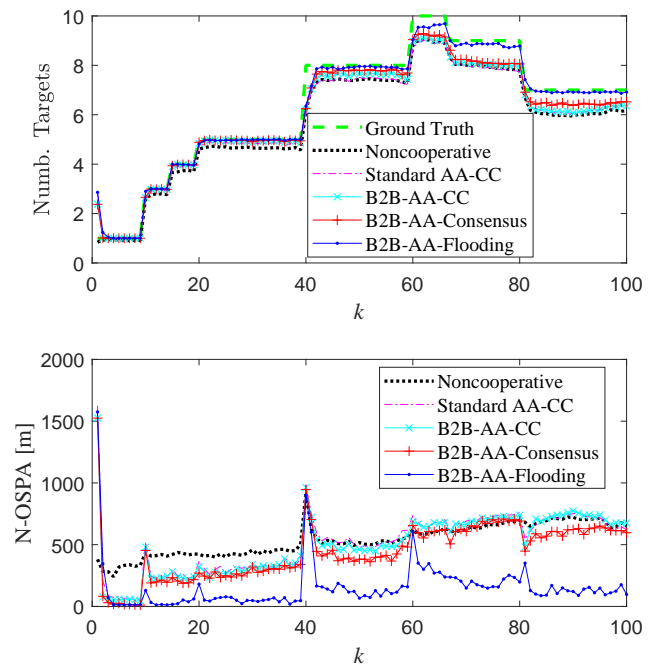

Fig. 7. Average estimated number of targets and N-OSPA of different filters over time in the 2 nd scenario.

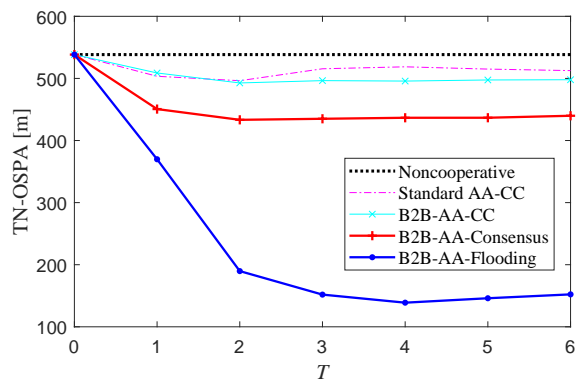

Fig. 8. TN-OSPAs of different filters over time for different numbers $T$ of communication iterations in the 2 nd scenario. 


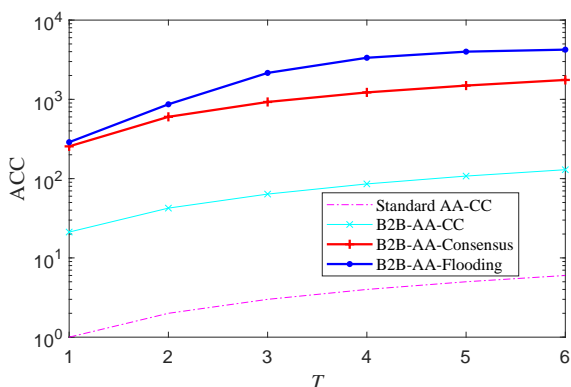

Fig. 9. ACCs of different filters for different numbers $T$ of communication iterations in the 2 nd scenario.

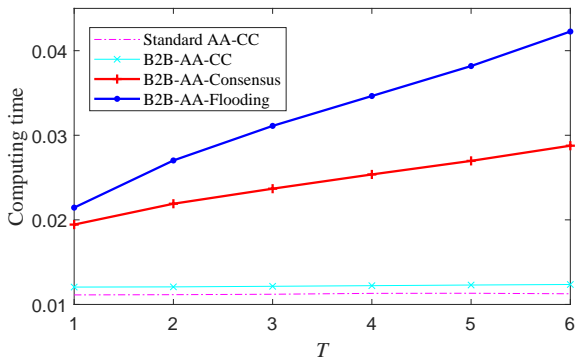

Fig. 10. Computing time for each filtering iteration of different filters for different numbers $T$ of communication iterations in the 2 nd scenario.

\section{APPENDIX}

Given two GCs $\left(\hat{\mathbf{x}}_{i}, \boldsymbol{\Sigma}_{i}\right)$ weighted as $\omega_{i} \geq 0, i=1,2$, respectively, the mixture merging scheme [55] fuses them into a single GC $\left(\hat{\mathbf{x}}_{\mathrm{M}}, \boldsymbol{\Sigma}_{\mathrm{M}}\right)$ with weight $\omega_{\mathrm{M}}$, given by

$$
\begin{gathered}
\omega_{\mathrm{M}}=\omega_{1}+\omega_{2}, \\
\hat{\mathbf{x}}_{\mathrm{M}}=\frac{\omega_{1} \hat{\mathbf{x}}_{1}+\omega_{2} \hat{\mathbf{x}}_{2}}{\omega_{1}+\omega_{2}}, \\
\boldsymbol{\Sigma}_{\mathrm{M}}=\frac{\omega_{1} \tilde{\boldsymbol{\Sigma}}_{1}+\omega_{2} \tilde{\boldsymbol{\Sigma}}_{2}}{\omega_{1}+\omega_{2}},
\end{gathered}
$$

with $\tilde{\boldsymbol{\Sigma}}_{i}=\boldsymbol{\Sigma}_{i}+\left(\hat{\mathbf{x}}_{\mathrm{M}}-\hat{\mathbf{x}}_{i}\right)\left(\hat{\mathbf{x}}_{\mathrm{M}}-\hat{\mathbf{x}}_{i}\right)^{\mathrm{T}}$.

Lemma 3. The above merging scheme does not change the mean or variance of the concerning distribution.

Proof. We start by recalling that the mean and covariance of a distribution function are defined as $\boldsymbol{\mu} \triangleq \int_{\mathbb{R}^{d}} \mathbf{x} \bar{\nu}(\mathbf{x}) \mathrm{d} \mathbf{x}$ and $\boldsymbol{\Sigma} \triangleq \int_{\mathbb{R}^{d}}(\mathbf{x}-\boldsymbol{\mu})(\mathbf{x}-\boldsymbol{\mu})^{\mathrm{T}} \bar{\nu}(\mathbf{x}) \mathrm{d} \mathbf{x}$, respectively, where $\bar{\nu}(\mathbf{x}) \triangleq$ $\nu(\mathbf{x}) / \int_{\mathbb{R}^{d}} \nu\left(\mathbf{x}^{\prime}\right) \mathrm{d} \mathbf{x}^{\prime}$

Consider a GM of two GCs $\nu_{1}(\mathbf{x})=\omega_{1} \mathcal{N}\left(\mathbf{x} ; \boldsymbol{\mu}_{1}, \boldsymbol{\Sigma}_{1}\right)$ and $\nu_{2}(\mathbf{x})=\omega_{2} \mathcal{N}\left(\mathbf{x} ; \boldsymbol{\mu}_{2}, \boldsymbol{\Sigma}_{2}\right)$, whose joint distribution is simply $\nu_{\mathrm{GM}}(\mathbf{x})=\nu_{1}(\mathbf{x})+\nu_{2}(\mathbf{x})$, where $\omega_{1} \geq 0$ and $\omega_{2} \geq 0$. The mean of $\nu_{\mathrm{GM}}(\mathbf{x})$ is readily shown to be the weighted average of $\boldsymbol{\mu}_{1}$ and $\boldsymbol{\mu}_{2}$, i.e.,

$$
\boldsymbol{\mu}_{\mathrm{GM}}=\frac{\omega_{1} \boldsymbol{\mu}_{1}+\omega_{2} \boldsymbol{\mu}_{2}}{\omega_{1}+\omega_{2}}
$$

and the covariance of $\nu_{\mathrm{GM}}(\mathbf{x})$ is obtained as

$$
\begin{aligned}
\boldsymbol{\Sigma}_{\mathrm{GM}} & =\frac{\omega_{1}\left(\boldsymbol{\Sigma}_{1}+\boldsymbol{\mu}_{1} \boldsymbol{\mu}_{1}^{\mathrm{T}}\right)+\omega_{2}\left(\boldsymbol{\Sigma}_{2}+\boldsymbol{\mu}_{2} \boldsymbol{\mu}_{2}^{\mathrm{T}}\right)}{\omega_{1}+\omega_{2}}-\boldsymbol{\mu}_{\mathrm{GM}} \boldsymbol{\mu}_{\mathrm{GM}}^{\mathrm{T}} \\
& =\frac{\omega_{1} \boldsymbol{\Sigma}_{1}+\omega_{2} \boldsymbol{\Sigma}_{2}}{\omega_{1}+\omega_{2}}+\boldsymbol{\Sigma}_{\Delta}\left(\omega_{1}, \omega_{2}\right),
\end{aligned}
$$

with $\boldsymbol{\Sigma}_{\Delta}\left(\omega_{1}, \omega_{2}\right) \triangleq \frac{\omega_{1} \omega_{2}}{\left(\omega_{1}+\omega_{2}\right)^{2}}\left(\boldsymbol{\mu}_{1}-\boldsymbol{\mu}_{2}\right)\left(\boldsymbol{\mu}_{1}-\boldsymbol{\mu}_{2}\right)^{\mathrm{T}}$.
On the other hand, applying the merging scheme as given in (39),(40) and (41) to $\nu_{1}(\mathbf{x})$ and $\nu_{2}(\mathbf{x})$ yields a single GC $\nu_{\mathrm{M}}(\mathbf{x})=\left(\omega_{1}+\omega_{2}\right) \mathcal{N}\left(\mathbf{x} ; \boldsymbol{\mu}_{\mathrm{M}}, \boldsymbol{\Sigma}_{\mathrm{M}}\right)$, whose mean (c.f. (40)) and covariance (c.f. (41)) are given by, respectively,

$$
\begin{gathered}
\boldsymbol{\mu}_{\mathrm{M}}=\frac{\omega_{1} \boldsymbol{\mu}_{1}+\omega_{2} \boldsymbol{\mu}_{2}}{\omega_{1}+\omega_{2}}, \\
\boldsymbol{\Sigma}_{\mathrm{M}}=\frac{\omega_{1}\left(\boldsymbol{\Sigma}_{1}+\boldsymbol{e}_{1}\right)+\omega_{2}\left(\boldsymbol{\Sigma}_{2}+\boldsymbol{e}_{2}\right)}{\omega_{1}+\omega_{2}},
\end{gathered}
$$

where $\boldsymbol{e}_{i} \triangleq\left(\boldsymbol{\mu}_{i}-\boldsymbol{\mu}_{\mathrm{M}}\right)\left(\boldsymbol{\mu}_{i}-\boldsymbol{\mu}_{\mathrm{M}}\right)^{\mathrm{T}}, i=1,2$. It is easy to be verified that, $\mu_{\mathrm{M}}=\mu_{\mathrm{GM}}$ and $\Sigma_{\mathrm{M}}=\Sigma_{\mathrm{GM}}$.

\section{REFERENCES}

[1] Y. Bar-Shalom, P. Willett, and X. Tian, Tracking and Data Fusion: A Handbook of Algorithms. YBS Publishing, 2011.

[2] B.-N. Vo, M. Mallick, Y. Bar-shalom, S. Coraluppi, R. Osborne, R. Mahler, and B.-T. Vo, Multitarget Tracking. John Wiley \& Sons, Inc., 2015.

[3] F. Meyer, T. Kropfreiter, J. L. Williams, R. Lau, F. Hlawatsch, P. Braca, and M. Z. Win, "Message passing algorithms for scalable multitarget tracking," Proc. IEEE, vol. 106, no. 2, pp. 221-259, Feb. 2018.

[4] R. P. S. Mahler, Statistical Multisource-Multitarget Information Fusion. Norwood, MA, USA: Artech House, 2007.

[5] —, Advances in Statistical Multisource-Multitarget Information Fusion. Norwood, MA, USA: Artech House, 2014.

[6] — "Multitarget Bayes filtering via first-order multitarget moments," IEEE Trans. Aerosp. Electron. Syst., vol. 39, no. 4, pp. 1152-1178, Oct. 2003.

[7] B.-N. Vo, S. Singh, and A. Doucet, "Sequential Monte Carlo methods for multitarget filtering with random finite sets," IEEE Trans. Aerosp. Electron. Syst., vol. 41, no. 4, pp. 1224-1245, Oct. 2005.

[8] B.-N. Vo and W. K. Ma, "The Gaussian mixture probability hypothesis density filter," IEEE Trans. Signal Process., vol. 54, no. 11, pp. 40914104, Nov. 2006

[9] B. Vo, B. Vo, and A. Cantoni, "Analytic implementations of the cardinalized probability hypothesis density filter," IEEE Trans. Signal Process., vol. 55, no. 7, pp. 3553-3567, July 2007.

[10] _ , "The cardinality balanced multi-target multi-Bernoulli filter and its implementations," IEEE Trans. Signal Process., vol. 57, no. 2, pp. 409-423, Feb. 2009.

[11] C. Ouyang, H. Ji, and C. Li, "Improved multi-target multi-Bernoulli filter," IET Radar, Sonar Navigation, vol. 6, no. 6, pp. 458-464, Jul. 2012.

[12] J. L. Williams, "Marginal multi-Bernoulli filters: RFS derivation of MHT, JIPDA, and association-based MeMBer," IEEE Trans. Aerosp. Electron. Syst., vol. 51, no. 3, pp. 1664-1687, Jul. 2015.

[13] K. Granström, P. Willett, and Y. Bar-Shalom, "Approximate multihypothesis multi-Bernoulli multi-object filtering made multi-easy," IEEE Trans. Signal Process., vol. 64, no. 7, pp. 1784-1797, Apr. 2016.

[14] C. Chong, S.Mori, and K. Chang, "Distributed multitarget multisensory tracking," in Multitarget Multisensor Tracking: Advanced applications, Y. Bar-Shalom, Ed. Artech House, 1990.

[15] K. C. Chang, R. K. Saha, and Y. Bar-Shalom, "On optimal track-totrack fusion," IEEE Trans. Aerosp. Electron. Syst., vol. 33, no. 4, pp. 1271-1276, Oct. 1997.

[16] A. Saucan, M. J. Coates, and M. Rabbat, "A multisensor multi-Bernoulli filter,' IEEE Trans. Signal Process., vol. 65, no. 20, pp. 5495-5509, Oct. 2017.

[17] T. Li and F. Hlawatsch, "A distributed particle-PHD filter using arithmetic-average fusion of Gaussian mixture parameters," Inf. Fusion, Dec. 2018, submitted. Preprint: arXiv:1712.06128v2.

[18] T. Li, M. Mallick, and Q. Pan, "A parallel filtering-communication based cardinality consensus approach for real-time distributed PHD filtering," IEEE Trans. Aerosp. Electron. Syst., Submitted, June 2019.

[19] J. K. Uhlmann, "Dynamic map building and localization: New theoretical foundations," Ph.D. dissertation, University of Oxford, UK, 1995.

[20] T. Bailey, S. Julier, and G. Agamennoni, "On conservative fusion of information with unknown non-Gaussian dependence," in Proc. FUSION 2012, Singapore, Jul. 2012, pp. 1876-1883.

[21] L. Xiao and S. Boyd, "Fast linear iterations for distributed averaging," Syst. Control. Lett., vol. 53, no. 1, pp. 65-78, 2004. 
[22] L. Xiao, S. Boyd, and S. Lall, "A scheme for robust distributed sensor fusion based on average consensus," in Proc. IPSN 2005, Los Angeles, California, CA, USA, Apr. 2005, pp. 63-70.

[23] R. Olfati-Saber, J. A. Fax, and R. M. Murray, "Consensus and cooperation in networked multi-agent systems," Proc. IEEE, vol. 95, no. 1, pp. 215-233, Jan. 2007.

[24] W. Ren, R. W. Beard, and E. M. Atkins, "Information consensus in multivehicle cooperative control," IEEE Contr. Syst. Mag., vol. 27, no. 2, pp. 71-82, Apr. 2007.

[25] T. Li, H. Fan, J. García, and J. M. Corchado, "Second-order statistics analysis and comparison between arithmetic and geometric average fusion: Application to multi-sensor target tracking," Inf. Fusion, vol. 51, pp. $233-243,2019$.

[26] R. P. S. Mahler, "Toward a theoretical foundation for distributed fusion," in Distributed Data Fusion for Network-Centric Operations, D. Hall, C.Y. Chong, J. Llinas, and M. Liggins, Eds. Boca Raton, FL, USA: CRC Press, 2012, pp. 199-224.

[27] D. Clark, S. Julier, R. Mahler, and B. Ristic, "Robust multi-object sensor fusion with unknown correlations," in Proc. SSPD 2010, London, UK, Sep. 2010.

[28] G. Battistelli, L. Chisci, C. Fantacci, A. Farina, and A. Graziano, "Consensus CPHD filter for distributed multitarget tracking," IEEE J. Sel. Topics Signal Process., vol. 7, no. 3, pp. 508-520, Jun. 2013.

[29] M. Üney, D. E. Clark, and S. J. Julier, "Distributed fusion of PHD filters via exponential mixture densities," IEEE J. Sel. Topics Signal Process., vol. 7, no. 3, pp. 521-531, Jun. 2013.

[30] M. Üney, J. Houssineau, E. Delande, S. J. Julier, and D. E. Clark, "Fusion of finite set distributions: Pointwise consistency and global cardinality," IEEE Trans. Aerosp. Electron. Syst., vol. 55, no. 6, pp. 2759-2773, Dec. 2019

[31] M. Gunay, U. Orguner, and M. Demirekler, "Chernoff fusion of Gaussian mixtures based on sigma-point approximation," IEEE Trans. Aerosp. Electron. Syst., vol. 52, no. 6, pp. 2732-2746, Dec. 2016.

[32] T. Li, J. Corchado, and S. Sun, "Partial consensus and conservative fusion of Gaussian mixtures for distributed PHD fusion," IEEE Trans. Aerosp. Electron. Syst., vol. 55, no. 5, pp. 2150-2163, Oct. 2019.

[33] G. Battistelli, L. Chisci, C. Fantacci, N. Forti, A. Farina, and A. Graziano, "Distributed peer-to-peer multitarget tracking with association-based track fusion," in Proc. FUSION 2014, Salamanca, Spain, Jul. 2014, pp. 1-7.

[34] J. Y. Yu, M. Coates, and M. Rabbat, "Distributed multi-sensor CPHD filter using pairwise gossiping," in Proc. IEEE ICASSP 2016, Shanghai, China, Mar. 2016, pp. 3176-3180.

[35] L. Gao, G. Battistelli, and L. Chisci, "Multiobject fusion with minimum information loss," IEEE Signal Process. Lett., Dec. 2019.

[36] W. Yi, M. Jiang, S. Li, and B. Wang, "Distributed sensor fusion for RFS density with consideration of limited sensing ability," in Proc. FUSION 2017, Xi’an, China, Jul. 2017.

[37] T. Li, V. Elvira, H. Fan, and J. M. Corchado, "Local-diffusion-based distributed SMC-PHD filtering using sensors with limited sensing range," IEEE Sensors J., vol. 19, no. 4, pp. 1580-1589, Feb. 2019

[38] R. L. Streit, "Multisensor multitarget intensity filter," in Proc. FUSION 2008, Cologne, Germany, Jun. 2008.

[39] T. Li, J. Corchado, and S. Sun, "On generalized covariance intersection for distributed PHD filtering and a simple but better alternative," in Proc. FUSION 2017, Xi'an, China, Jul. 2017, pp. 808-815.

[40] A. K. Gostar, R. Hoseinnezhad, and A. Bab-Hadiashar, "CauchySchwarz divergence-based distributed fusion with Poisson random finite sets," in Proc. ICCAIS 2017, Chiang Mai, Thailand, Oct. 2017, pp. 112 116.

[41] T. Li, F. Hlawatsch, and P. M. Djuríc, "Cardinality-consensus-based PHD filtering for distributed multitarget tracking," IEEE Signal Process. Lett., vol. 26, no. 1, pp. 49-53, Jan. 2019.

[42] T. Li, Z. Liu, and Q. Pan, "Distributed Bernoulli filtering for target detection and tracking based on arithmetic average fusion," IEEE Signal Process. Lett., vol. 26, no. 12, pp. 1812-1816, Dec. 2019.

[43] K. Da, T. Li, Y. Zhu, and Q. Fu, "A computationally efficient approach for distributed sensor localization and multi- target tracking," IEEE Commun. Lett., 2019, published online. Doi:10.1109/LCOMM.2019.29548881903.04239.

[44] N. Lehrer, O. Tslil, and A. Carmi, "Log-linear Chernoff fusion for distributed particle filtering," in Proc. FUSION 2019, Ottawa, Canada, Jul. 2019.

[45] K. Da, T. Li, Y. Zhu, H. Fan, and Q. Fu, "Kullback-Leibler averaging for multitarget density fusion," in Proc. DCAI 2019, Avila, Spain, Jun. 2019, pp. 253-261.

[46] M. R. Fréchet, "Les éléments aléatoires de nature quelconque dans un espace distancié," Annales de l'institut Henri Poincaré, vol. 10, no. 4, pp. 215-310, 1948

[47] F. Nielsen and R. Bhatia, Matrix Information Geometry. Springer Berlin Heidelberg, 2012.

[48] M. Jiang, W. Yi, R. Hoseinnezhad, and L. Kong, "Distributed multisensor fusion using generalized multi-Bernoulli densities," in Proc. FUSION 2016, July 2016, pp. 1332-1339.

[49] B. Wang, W. Yi, R. Hoseinnezhad, S. Li, L. Kong, and X. Yang, "Distributed fusion with multi-Bernoulli filter based on generalized covariance intersection," IEEE Trans. Signal Process., vol. 65, no. 1, pp. 242-255, Jan. 2017

[50] W. Yi, S. Li, B. Wang, R. Hoseinnezhad, and L. Kong, "Computationally efficient distributed multi-sensor fusion with multi-Bernoulli filter," IEEE Trans. Signal Process., pp. 1-1, 2019.

[51] T. Li, J. Corchado, and J. Prieto, "Convergence of distributed flooding and its application for distributed Bayesian filtering," IEEE Trans. Signal Inf. Process. Netw., vol. 3, no. 3, pp. 580-591, Sep. 2017.

[52] L. Xiao, S. Boyd, and S.-J. Kim, "Distributed average consensus with least-mean-square deviation," J Parallel. Dist. Comput., vol. 67, no. 1, pp. $33-46,2007$.

[53] A. E. Abbas, "A Kullback-Leibler view of linear and log-linear pools," Decision Analysis, vol. 6, no. 1, pp. 25-37, 2009.

[54] C. Dwork, R. Kumar, M. Naor, and D. Sivakumar, "Rank aggregation methods for the web," in Proc. 10th $I C W W W$. New York, NY, USA: ACM, 2001, pp. 613-622.

[55] D. J. Salmond, "Mixture reduction algorithms for target tracking in clutter," in Proc. SPIE, vol. 1305, 1990, pp. 434-445.

[56] T. Li, J. M. Corchado, and H. Chen, "Distributed flooding-thenclustering: A lazy networking approach for distributed multiple target tracking," in Proc. FUSION 2018, Jul. 2018, pp. 2415-2422.

[57] T. Li, J. Prieto, H. Fan, and J. M. Corchado, "A robust multi-sensor PHD filter based on multi-sensor measurement clustering," IEEE Commun Lett., vol. 22, no. 10, pp. 2064-2067, Oct 2018.

[58] J. Munkres, "Algorithms for the assignment and transportation problems," J. Soc. Ind. Appl. Math., vol. 5, no. 1, pp. 32-38, 1957.

[59] D. Schuhmacher, B.-T. Vo, and B.-N. Vo, "A consistent metric for performance evaluation of multi-object filters," IEEE Trans. Signal Process., vol. 56, no. 8, pp. 3447-3457, Aug. 2008.

[60] A. De Freitas, L. Mihaylova, A. Gning, M. Schikora, M. Ulmke, D. Angelova, and W. Koch, "A box particle filter method for tracking multiple extended objects," IEEE Trans. Aerosp. Electr. Syst., vol. 55, no. 4, pp. 1640-1655, Aug. 2019.

[61] A. H. Sayed, P. M. Djuri, and F. Hlawatsch, "Chapter 6 - distributed Kalman and particle filtering," in Cooperative and Graph Signal Processing, P. M. Djurić and C. Richard, Eds. Academic Press, 2018, pp. $169-207$.

[62] S. Deb, M. Yeddanapudi, K. Pattipati, and Y. Bar-Shalom, "A generalized S-D assignment algorithm for multisensor-multitarget state estimation," IEEE Trans. Aeros. Electron. Syst., vol. 33, no. 2, pp. 523538, Apr. 1997.

[63] S. He, H. Shin, and A. Tsourdos, "Track-oriented multiple hypothesis tracking based on tabu search and Gibbs sampling," IEEE Sensors J., vol. 18, no. 1, pp. 328-339, Jan. 2018.

[64] B. Vo and B. Vo, "Labeled random finite sets and multi-object conjugate priors," IEEE Trans. Signal Process., vol. 61, no. 13, pp. 3460-3475, Jul. 2013.

[65] — - "A multi-scan labeled random finite set model for multi-object state estimation," IEEE Trans. Signal Process., vol. 67, no. 19, pp. 49484963, Oct. 2019. 